\title{
Adapting the Ranging Algorithm to the Positioning Technique in UWB Sensor Networks
}

\author{
Luca Reggiani \\ Dip. di Elettronica ed Informazione \\ Politecnico di Milano \\ 20133 Milano, Italy \\ E-mail: reggiani@elet.polimi.it
}

\author{
Mats Rydström, Erik G. Ström and Arne Svensson \\ Dept. of Signals and Systems \\ Chalmers University of Technology \\ SE-412-96 Göteborg, Sweden \\ E-mail: $\{$ d98mats, estrom, arnes\}@chalmers.se
}

\begin{abstract}
Ultra-Wideband (UWB) is an emerging technology for short-range wireless communications. Due to the high bandwidth of UWB signals, accurate ranging and positioning is possible, which is one of many reasons why UWB is a candidate physical layer for another emerging research area; that of wireless sensor networks (WSNs). In this work, we argue that the ranging algorithm design should be made with the characteristics of the positioning algorithm in mind. In considering ranging and positioning in parallel, we are able to construct an estimator of range that is tailored to the positioning algorithm, thereby achieving robustness to non-line-of-sight (NLOS) effects, reasonable overall complexity, and good performance in terms of positioning error. The analysis and simulations in this work are based on the channel models adopted by the IEEE 802.15.4a working group, and highlight the benefits and drawbacks of the proposed approach.
\end{abstract}

\section{INTRODUCTION}

In a wireless sensor network (WSN), information about the position of individual nodes, either absolute or in relation to other nodes in the network, is often crucial for a successful fulfilment of the network purpose. One of the candidate physical layers for this type of network is ultra-wideband impulse radio (UWB-IR) [1], that potentially combine low power consumption, immunity to multipath fading, low probability of intercept, and, supposedly, low cost. Due to the large bandwidth employed by UWB-IR, accurate propagation delay based ranging between nodes is feasible [2]. Such distance estimates may then be used to estimate the positions of nodes in the network. However, many obstacles need to be overcome before a successful WSN positioning system can be realized; (a) Most WSNs will be asynchronous, meaning that there is no available global time reference accurate enough for direct propagation delay distance estimation. (b) The channel statistics must, in most cases, be assumed unknown at the receiver. (c) There are tight constraints on energy consumption, cost and size, limiting the allowable complexity. (d) Devices will most likely not be homogenous, i.e., different types of receiver front-ends will be present in the network. (e) A given percentage of channels will be non-line-of-sight (NLOS), adding a considerable positive bias to most distance estimators.

An extensive literature exist on the topic of ranging and positioning in UWB systems. In [3], Lee and Scholtz describe a correlator-based range estimator of reasonable complexity.

\footnotetext{
${ }^{1}$ This work was supported in part by European Union FP-6 Project NEWCOM, Contract IST NoE 507325, and in part by Vinnova project no. 2003-02803.
}

The same authors also propose an estimator based on a maximum likelihood (ML) approach [4]. This ML-type estimator provides good performance, but at the expense of considerable complexity. In [5], a comparative study of more advanced algorithms for determining time of arrival of UWB signals for different receiver architectures was recently presented. However, the work in [5] differs from the work presented here in several aspects, the assumption of prior information about the channel being the most significant. For an overview of current work, including both ranging and positioning algorithms, the reader is referred to [2], and references cited therein.

In this paper, we argue that improvements in both positioning accuracy and robustness are possible if the error characteristics of the ranging scheme can be adapted and matched to the characteristics of the positioning scheme. Towards this end, a novel ranging algorithm, that operates without a global time reference and assumes no prior channel knowledge is designed. The algorithm does not output a single distance estimate, as most proposed algorithms in the literature do, but several distance estimates with associated likelihoods. Using these "soft" distance estimates, we are able to tune the error distribution to fit with a given positioning algorithm. The positioning algorithm used as an example here is based on projections onto convex sets (POCS) [6], a method that is remarkably resilient to arbitrarily large positive errors in distance estimates, but suffers if large negative errors in distance estimates occur. Hence, the distance estimator is tuned to output a minimum of estimates with negative errors, at the expense of intermittent large positive errors and higher variance. Other positioning algorithms, such as leastsquares approaches, does not have this peculiarity, and thus the distance estimator is tuned differently.

The overall organization of this work is as follows: Section II describes the network scenario and the network nodes. Section III is dedicated to the derivation of the ranging algorithm, while Sec. IV briefly presents the localization algorithm. Finally, a short evaluation in terms of numerical results is given in Sec. V, and the paper is concluded in Sec. VI.

\section{SYSTEM MODEL}

The network under consideration is an asynchronous UWBIR sensor network composed of a fixed number $M$ of static nodes distributed over a delimited area, and with twodimensional coordinates $\left\{\mathbf{x}_{i}\right\}_{i=1}^{M}$. We assume the position of a subset of nodes (sometimes referred to as beacons) are known 
a-priori, but a generalization of the proposed system to a network without beacons is straight forward. The WSN lacks a global clock reference exact enough for one-way ranging, and nodes have no a-priori channel state information. The packet transmission in the network is totally uncoordinated, and each transmitter uses a node-specific and globally known preamble sequence for medium access. An alternative not considered here would be the use of a common preamble, perhaps with the addition of coordinated transmissions. This would alter interference conditions somewhat, but not change the main conclusions. Each transmitted packet is time-stamped with respect to the local clock at the transmitting node, and all, or a subset of nodes cooperatively estimate the unknown positions. It is assumed that the ranging and position estimation is repeated periodically, and we will only consider the first such period, i.e., network start-up. Of course, as time evolves, position estimates may be refined and tracked if nodes are moving, but this aspect is not considered here.

\section{A. UWB signal model}

The baseband signal $s_{j}(t)$, generated by the $j$ th node, comprises a preamble of length $L_{P R}$ frames and a data payload of $L_{D}$ frames, i.e.,

$$
\begin{aligned}
s_{j}(t)= & \sum_{k=0}^{L_{P R}-1} \bar{a}_{j, k} w\left(t-k T_{f}-\bar{c}_{j, k} T_{c}\right) \\
& +\sum_{k=L_{P R}}^{L_{P R}+L_{D}-1} a_{j, k} w\left(t-k T_{f}-c_{j, k} T_{c}\right),
\end{aligned}
$$

where $w(t)$ is a root-raised-cosine pulse waveform, $T_{f}$ is the UWB-IR frame time [1], and $T_{c}$ is the chip time. Each frame consists of an integer number $N_{f}=T_{f} / T_{c}$ of pulse slots, and each pulse is amplitude modulated (PAM) with polarity \pm 1 . The PAM symbol sequences $\left\{\bar{a}_{j, k}\right\}_{k=0}^{L_{\mathrm{PR}}-1}$, and $\left\{a_{j, k}\right\}_{k=L_{\mathrm{PR}}}^{L_{P R}+L_{D}-1}$ for preamble and data, respectively, are chosen randomly in this work. The time-hopping pseudo-noise (PN) sequences of node $j$, for preamble and data, respectively, is represented by $\left\{\bar{c}_{j, k}\right\}_{k=0}^{L_{\mathrm{PR}}-1}$, and $\left\{c_{j, k}\right\}_{k=L_{\mathrm{PR}}}^{L_{P R}+L_{D}-1}$. As mentioned above, the preamble sequences can in some scenarios be assumed equal for all $j$. The pulse has a $-3 \mathrm{~dB}$ bandwidth of $494 \mathrm{MHz}$, the roll-off factor is 0.25 , and the system under consideration operates around an RF carrier at $f_{0}=3.952 \mathrm{GHz}$. This choice is due to the recent IEEE 802.15.4a standardization trends, in which the mandatory signal $-3 \mathrm{~dB}$ bandwidth is $494 \mathrm{MHz}$ and the UWB-IR transmission is not carrierless but centered around one of several equispaced carriers. We observe that the expected distance resolution of the system turns out to be about $0.6 \mathrm{~m}$. All simulations are made at baseband and in discrete time, using a complex baseband-equivalent channel model adopted by the IEEE 802.15.4a working group. This channel model is a low-pass filtered tapped delay-line, where signal components arrive at the receiver in independent clusters. After passing the channel, the signal is affected by complex additive white Gaussian noise (CAWGN) with zero mean and unknown variance. The modeling and characteristics of the employed channel model are described in detail in [7].

\section{B. Receiver architectures}

The receiver architecture can either be built on a matched filter or an energy detector front-end, both sampled at $f_{s}=$ $1 / T_{c} \mathrm{~Hz}$. The receiver detects the presence of a given preamble sequence through correlating the sampled front-end output with the known PN sequences. The output of the correlator is squared, and the location in time of the strongest peak is denoted $T_{0}$.

It should be noted that the sampling instants are controlled by the data receiver, and not by the ranging algorithm. Thus, the ranging algorithm assumes random chip-spaced sampling. Also, since the algorithms proposed in this work operate only on the chip-spaced correlator output samples, only minor adjustments is needed if the front-end of the receiver is changed.

\section{RANGING ALGORITHM}

The ranging algorithm operates on a sequence of squared correlator outputs. Henceforth, a squared correlator sample at discrete time-offset $k$ will be denoted $y_{k}$. Here, we assume a matched filter front-end, but similar derivations result if an energy detector front-end is assumed.

First, an estimate of the variance $\sigma_{n}^{2}$ of the CAWGN in the correlator output is obtained. Under the assumption that a majority of correlator outputs contain only noise (sparse traffic), we have $\hat{\sigma}_{n}^{2}=\frac{1}{N} \sum_{k=1}^{N} y_{k}$, where $N$ is the correlator total output length. We assume all interference is included in the CAWGN. Thus, if there is no signal-component at offset $k, y_{k}$ follows an exponential distribution parameterized by $\sigma_{n}^{2}$. Therefore, using $\hat{\sigma}_{n}^{2}$, a fixed probability of false alarm $P_{\mathrm{FA}}$, and prior knowledge about the preamble PN-sequence autocorrelation function $\Psi_{\mathrm{PN}}$, a signal detection threshold given by $\theta=\max \left(-\hat{\sigma}_{\Psi_{n}}^{2} \ln \left(P_{\mathrm{FA}}\right), \rho y_{T_{0} / T_{c}}\right)$, can be derived, where $y_{T_{0} / T_{c}}$ is the peak correlator output (at $T_{0}$ ), and the factor $\rho=$ $\max _{n \neq 0} \frac{\Psi_{\mathrm{PN}}(n)}{\Psi_{\mathrm{PN}}(0)}$ is the ratio between the second strongest value of the known PN preamble sequence autocorrelation function $\Psi_{\mathrm{PN}}$ and its maximum value; this lower bound on $\theta$ limits false detections due to imperfections in the PN sequences, which mainly occur at high signal-to-interference-and-noise ratios (SINR). For low $P_{\mathrm{FA}}$, squared correlator samples in $\left\{y_{k}\right\}$ exceeding $\theta$ are likely to contain signal in addition to noise, and will henceforth be denoted $\left\{s_{i}\right\}_{i=1}^{N_{\tau}}$, with corresponding delays $\left\{\tau_{i}\right\}_{i=1}^{N_{\tau}}, N_{\tau}$ denoting the number of detected paths, $\left\{s_{i}\right\}_{i=1}^{N_{\tau}} \subseteq\left\{y_{k}\right\}_{k=1}^{N}$, and we assume $N_{\tau} \ll N$.

The search for the first path is now confined to a window of tunable duration preceding $T_{0}$. The motivation for this being that any peaks following the strongest peak is unlikely to correspond to the line-of-sight (LOS) signal path, and processing them would add considerably to the complexity. The window length used here is given by $T_{\mathrm{RW}}=\max \left(\lambda \hat{\tau}_{d}, \tau_{\min }\right)$ seconds, where $\lambda$ is a tuning parameter, $\tau_{\min }$ is for avoiding too short windows, and $\hat{\tau}_{d}$ is an estimate of the channel RMS delayspread. This estimate is given by

$$
\hat{\tau}_{d}=\sqrt{\frac{1}{N_{\tau} \overline{s_{i}}} \sum_{i} s_{i}\left(\tau_{i}-\left\langle\tau_{i}\right\rangle\right)^{2}},
$$


where $\left\langle\tau_{i}\right\rangle=\left(N_{\tau} \overline{s_{i}}\right)^{-1} \sum_{i} s_{i} \tau_{i}$, and $\overline{s_{i}}$ denotes the average of $\left\{s_{i}\right\}$ taken over all $i$. The windowed sample-set is therefore $\left[y_{\left(\left\lceil\left(T_{0}-T_{\mathrm{RW}}\right) / T_{c}\right\rceil\right)}, \ldots, y_{\left(T_{0} / T_{c}\right)}\right]$, where $\lceil a\rceil$ denotes the smallest integer greater than or equal to $a$.

Now, for each $y_{k}$ in the search window, we wish to assign an approximate probability $l_{n}\left(y_{k}\right)$ that the sample corresponds to noise only, and also an approximate probability $l_{s}\left(y_{k}\right)$ that there is a signal path at the given offset. As stated above, under the hypothesis $\mathcal{H}_{0}$ that there is only noise, the exponential PDF is a justifiable measure of noise only probability. Thus $l_{n}\left(y_{k}\right)=\left(1 / \hat{\sigma}_{n}^{2}\right) \exp \left(-y_{k} / \hat{\sigma}_{n}^{2}\right)$.

Since we, in order to keep derivations general, assume no prior channel information whatsoever, it is difficult to accurately model $y_{k}$ under the hypothesis $\mathcal{H}_{1}$ that a signalpath is present. However, simulation results show that even a very coarse approximation of the distribution of the $y_{k}$ 's under $\mathcal{H}_{1}$ gives better end results than if only $l_{n}\left(y_{k}\right)$ is used. Hence, we assume that the channel response has a rectangular power-delay profile locally around $T_{0}$, caused by received signal components that are uncorrelated and approximately follow a complex Gaussian distribution of mean $\mu$ and variance $\sigma_{c}^{2}$. With this crude approximation, $y_{k}$ is modelled by a noncentral $\chi_{2}^{2}$ distribution with unknown non-centrality parameter $a^{2}=2 \mu^{2}$, and underlying Gaussian variance $\sigma_{s}^{2}=\sigma_{c}^{2}+\sigma_{n}^{2}$. Thus,

$l_{s}\left(y_{k}\right)=p\left(y_{k} \mid \mathcal{H}_{1}\right)=\frac{1}{2 \sigma_{s}^{2}} \exp \left(-\frac{y_{k}+a^{2}}{2 \sigma_{s}^{2}}\right) I_{0}\left(\frac{\sqrt{a^{2} y_{k}}}{\sigma_{s}^{2}}\right)$,

where $I_{0}$ is the modified Bessel function of zero order. Parameters $\mu^{2}$ and $\sigma_{c}^{2}$ can be estimated, e.g., using a method of moments approach [8] as $\hat{\mu}^{2}=(1 / 2) \sqrt{{\overline{s_{i}}}^{2}-\left(1 / N_{\tau}\right) \sum_{i=1}^{N_{\tau}}\left(s_{i}-\overline{s_{i}}\right)^{2}}$ and $\hat{\sigma}_{c}^{2}=(1 / 2) \max \left(\left(\overline{s_{i}}-2 \hat{\mu}^{2}\right), 0\right)$. It should be noted that, when estimating $\mu^{2}$ and $\sigma_{c}^{2}$, the number of detected paths can be limited to the strongest $N_{\max }$ detected peaks, which will lower complexity at low SINR. Note also that, when we have a single detected peak, i.e., $N_{\tau}=1$, (1) reduces to a $\chi_{2}^{2}$ distribution with $a^{2}=s_{1}$ and $\sigma_{s}^{2}=\hat{\sigma}_{n}^{2}$.

Now, at each offset $k$, the approximate signal and noise probabilities are normalized, giving a probability of noise only as $\hat{p}_{n}\left(y_{k}\right)=l_{n}\left(y_{k}\right) /\left(l_{n}\left(y_{k}\right)+l_{s}\left(y_{k}\right)\right)$, that now considers both noise power and magnitude of detected paths. Using $\hat{p}_{n}$, we now wish to find a measure $l$ of the approximate probability that each correlator output sample $k$ represents the first signal path, i.e., the LOS component. Intuitively; (a) the LOS correlator peak is preceded by a number of noiseinduced peaks, (b) there is of course signal content in the peak, and (c) it will also likely be followed by other signal induced peaks, due to the clustered nature of the channel model. Our proposed measure is therefore given by $l(k)=$ $\left(1-\hat{p}_{n}\left(y_{k}\right)\right) \hat{p}_{f w}(k) \hat{p}_{b w}(k)$, where

$$
\hat{p}_{f w}(k)=\prod_{l \in w_{\mathrm{fw}}} \hat{p}_{n}\left(y_{l}\right), \text { and } \hat{p}_{b w}(k)=1-\prod_{l \in w_{\mathrm{bw}}} \hat{p}_{n}\left(y_{l}\right),
$$

and indices $k=\left\{\left\lceil\left(T_{0}-T_{\mathrm{RW}}\right) / T_{c}\right\rceil, \ldots, T_{0} / T_{c}\right\}, w_{\mathrm{fw}}=\{k-$ $\left.\left\lceil T_{\mathrm{RW}} / T_{c}\right\rceil, \ldots, k-1\right\}$, and $w_{\mathrm{bw}}=\left\{k+1, \ldots, k+\left\lceil T_{\mathrm{RW}} / T_{c}\right\rceil\right\}$. Thus, $\hat{p}_{f w}(k)$ is an approximation of the probability that no signal paths are present $T_{\mathrm{RW}}$ seconds before sample $k$ and $\hat{p}_{b w}(k)$ is an approximation of the probability that at least one signal path is present $T_{\mathrm{RW}}$ seconds after sample $k$. The addition of factor $\hat{p}_{b w}$ penalizes spurious single correlator peaks, that are not very probable in the considered channel model, but could very well be generated by a combination of interference and noise. An estimated LOS probability vector can now be formed at node $i$, by approximating LOS probabilities $l$ for all correlator output samples in the search window, normalizing to a unit sum, and stacking the outcome in a vector $\hat{\mathbf{p}}_{i}$.

All correlator peak delays $\boldsymbol{\tau}_{j, i}=\left[\tau_{j, i, 1}, \ldots, \tau_{j, i, N_{\tau, j}}\right]$, detected at node $i$ when correlating against the PN sequence from node $j$, corresponds to estimates of distance, albeit biased by unknown transmission and reception times with respect to a global clock reference. This bias may be cancelled by averaging two corresponding distance estimates. The reason this cancellation is possible is the sign-change in unknown clock-offsets $\Delta$ on the forward and reverse links [6], i.e., $\tau_{i, j}=\left\|\mathbf{x}_{i}-\mathbf{x}_{j}\right\| / c+\Delta_{i}-\Delta_{j}$ and $\tau_{j, i}=\left\|\mathbf{x}_{i}-\mathbf{x}_{j}\right\| / c+\Delta_{j}-\Delta_{i}$, where $c$ is the electromagnetic propagation velocity. Thus, the final distance estimate between nodes $i$ and $j$ is given by the vector $\hat{\mathbf{d}}_{i, j}=\left(\mathbf{1}_{N_{\tau, i}} \otimes \boldsymbol{\tau}_{i, j}+\boldsymbol{\tau}_{j, i} \otimes \mathbf{1}_{N_{\tau, j}}\right) c / 2$, where $\otimes$ denotes the Kronecker product and $\mathbf{1}_{N}$ is the $N$-length allones vector. Now, since some correlator offsets will be more likely than others of corresponding to the LOS component, some elements in $\hat{\mathbf{d}}_{i, j}$ will be more likely to correspond to the averaging of two LOS components than other elements. Since $\hat{\mathbf{d}}_{i, j}$ can be viewed as a sum of two random variables, it is in turn a random variable with a PDF given by the convolution of the two PDFs of the summand random variables. Therefore, after sorting and removing duplicate distance estimates from $\hat{\mathbf{d}}_{i, j}$, we can approximate the vector describing the probability that each element in $\hat{\mathbf{d}}_{i, j}$ is the average of two LOS correlator offsets by $\hat{\mathbf{p}}_{i, j}=\hat{\mathbf{p}}_{i} * \hat{\mathbf{p}}_{j}$, where $*$ denotes discrete convolution.

Given $\hat{\mathbf{d}}_{i, j}$ and $\hat{\mathbf{p}}_{i, j}$, a variety of estimators may be chosen from. For instance

$$
\begin{aligned}
\hat{d}_{i, j}^{l} & =\hat{\mathbf{p}}_{i, j}^{T} \hat{\mathbf{d}}_{i, j}, \\
\hat{d}_{i, j}^{m} & =\left\{\hat{d}_{i, j, k}: k=\arg \max _{q} \hat{p}_{i, j, q}\right\}, \\
\hat{d}_{i, j}^{t h}(t) & =\min _{k}\left\{\hat{d}_{i, j, k}: \sum_{n=1}^{k} \hat{p}_{i, j, n} \geq t\right\} .
\end{aligned}
$$

Of course, (2) is similar to the maximum a-posteriori estimator, while (3) is influenced by the maximum likelihood estimator. Both (2) and (3) are suited for positioning algorithms were negative and positive errors have the same effect, and a small error variance is sought. A robust algorithm where outliers is not a major problem could perhaps benefit from (3), while (2) would present fewer outliers. Estimator (4) is a thresholded estimator, aimed at limiting the probability of suffering a negative error. This type of estimator is especially suited for positioning algorithms based on POCS, described below. Some algorithms, for instance based on multiple-hypothesis tracking (MHT) or particle filter tracking, may even prefer the entire 
$\hat{\mathbf{d}}_{i, j}$ and $\hat{\mathbf{p}}_{i, j}$ vectors as input. It should be noted that the ranging algorithm does not control the chip-spaced sampling instants. Therefore, due to sampling mismatch with respect to the LOS component, there will be an irreducible error floor in all distance estimators.

Based on $\hat{\mathbf{p}}_{i, j}$, a measure of uncertainty or estimate of variance in the distance estimate is obtained. We call this measure the spread, $s_{i, j}$, of the distance estimate vector between nodes $i$ and $j$, and define it as

$$
s_{i, j}=\sum_{n} \hat{p}_{i, j, n}\left(\hat{d}_{i, j, n}-\hat{d}_{i, j}\right)^{2},
$$

where $\hat{d}_{i, j}$ can be any of the proposed estimators in (2)-(4). The spread is lower bounded by 0 , in which case only one estimate exists, with $\hat{\mathbf{p}}_{i, j, 1}=1$. As the number of likely LOS paths grow, the spread increases.

\section{Positioning ALGORIthm}

By design, the thresholded distance estimator in (4) aims for a positive bias, and has not been optimized for low variance. Instead, the design seeks to minimize the amount of large negative errors. The reason for this somewhat unconventional distance estimator is the recent availability of a positioning algorithm that is insensitive to intermittent but large positive distance errors. The positioning algorithm is based on the method of projections onto convex sets (POCS) [9]. The use of POCS for positioning in wireless sensor networks was, to the best of our knowledge, first proposed by Hero and Blatt in [10], and later extended in [6] to also include positioning of sensors outside the beacon perimeter. Below, due to space limitation, we only briefly introduce the POCS positioning algorithms. The reader is instead referred to [6], [9], [10] and references cited therein, for a more in-depth description and analysis of the positioning algorithm.

\section{A. Projection onto Convex sets}

The main difference between POCS and classical gradientbased approaches to positioning is that POCS does not operate on a global objective function, but instead sequentially projects a point in the plane onto convex sets that are formed from range estimates. Thus, POCS is not sensitive to objective function local minima, and, depending on the shape of the convex sets used, can also be made robust to intermittent but large positive errors in distance estimates. For instance, convex discs will only be inflated by a positive bias, and therefore not affect POCS iterations [6]. POCS is also easily distributed between nodes in the network. Thus, no central control or NLOS estimation procedure is needed.

The POCS method for distance estimates [9, ch. 5] is:

1. initialization $\mathbf{x}^{0}$ is arbitrary.

2. $\mathbf{x}^{\nu+1}=\mathbf{x}^{\nu}+\lambda_{\nu}\left[\mathcal{P}_{\mathcal{D}_{\iota(\nu)}}\left(\mathbf{x}^{\nu}\right)-\mathbf{x}^{\nu}\right]$, where $\left\{\lambda_{\nu}\right\}_{\nu=0}^{\infty}$ are relaxation parameters defined in, e.g., [6], $\mathcal{P}_{\mathcal{D}_{i}}(\cdot)$ is the projection function onto the $i$ th convex set $\mathcal{D}_{i}$, and $\iota(\nu)=\nu \bmod C$, where $C$ denotes the number of available convex sets.

In this work, two flavors of POCS have been used; circular and hybrid, both described in more detail in [6].

\section{Numerical Results}

All simulations have been carried out using the channel models for IEEE 802.15 LDR (Low Data Rate) systems [7]. The chosen channel models are CM1 and CM2, corresponding to a residential LOS and NLOS channel respectively. The network is composed of beacons and sensors equipped with matched filter front-ends (Sec. II-B). Each node uses a predefined transmission power of $-14.3 \mathrm{dBm}$. For each link, a channel realization was drawn from CM1 with probability $P_{\mathrm{LOS}}$, and from CM2 with probability $1-P_{\mathrm{LOS}}$. For LOS channel realizations, the path-loss exponent $\gamma_{\mathrm{LOS}}=2$, while for NLOS realizations $\gamma_{\mathrm{NLOS}}=3$. The reference path-loss was $34 \mathrm{~dB}$ at $d_{0}=1 \mathrm{~m}$. The variance of CAWGN was computed from a receiver noise figure of $7 \mathrm{~dB}$. All nodes transmit (uncoordinated) one packet containing their unique preamble, thereby enabling ranging, but also generating interference. The physical layer tuning parameters were empirically chosen as $\lambda=2, N_{\max }=10$, and $\tau_{\min }=6 T_{c}$. The POCS algorithms were run for a maximum of 40 iterations or until convergence.

\section{A. Ranging}

Four beacons were positioned at the corners of a square office-space with side $10 \mathrm{~m}$, and one sensor was located in the center of the space. There were no blocked channels, i.e., $P_{\text {LOS }}=1.0$. In Fig. 1, the cumulative-density-function (CDF) of the distance estimation error is plotted for preamble lengths of $L_{\mathrm{PR}}=16$ and $L_{\mathrm{PR}}=32$. The simulations were repeated 500 times, each with new noise and channel realizations. For comparison, the low-complexity estimator proposed in [3] was also implemented (henceforth denoted the comparison estimator), using the same search window as the proposed algorithm and a threshold optimized for the channel model under consideration. For the noise characteristics and set-up in this simulation, it was noted that estimators in (2) and (3) had very similar error CDFs, and therefore only (3) is plotted. The curve "jaggedness" is due to the discrete-time model of the system. We note that the estimator in (3) suffers from negative errors. This is due to the many noise and interference induced correlator peaks of relatively high amplitude preceding the peak at $T_{0}$, that are assigned non-zero probabilities, causing a large spread that in turn indicates bad quality. The comparison estimator is affected similarly, although there is no quality indicator here. Thus, at low SINR, neither of these estimators can be considered very appropriate for POCS. The thresholded estimator in (4) on the other hand, has a tendency to reject negative errors, trading them for larger positive errors. We also note that there is a considerable outage, especially for $L_{\mathrm{PR}}=16$, that occurs when one or both nodes on a link fail to detect the presence of a preamble, or when the resulting distance estimate is negative. If SINR is increased, it is seen that the estimators start to converge, and the outage decreases. At high SINR, i.e., large coding gains $\left(L_{\mathrm{PR}} \approx 128\right.$ for this scenario), estimators (2)-(4), and the comparison estimator show similar CDFs. Errors are then mainly caused by the random chip-spaced sampling (between $-0.3 \mathrm{~m}$ and $+0.3 \mathrm{~m}$ ). 


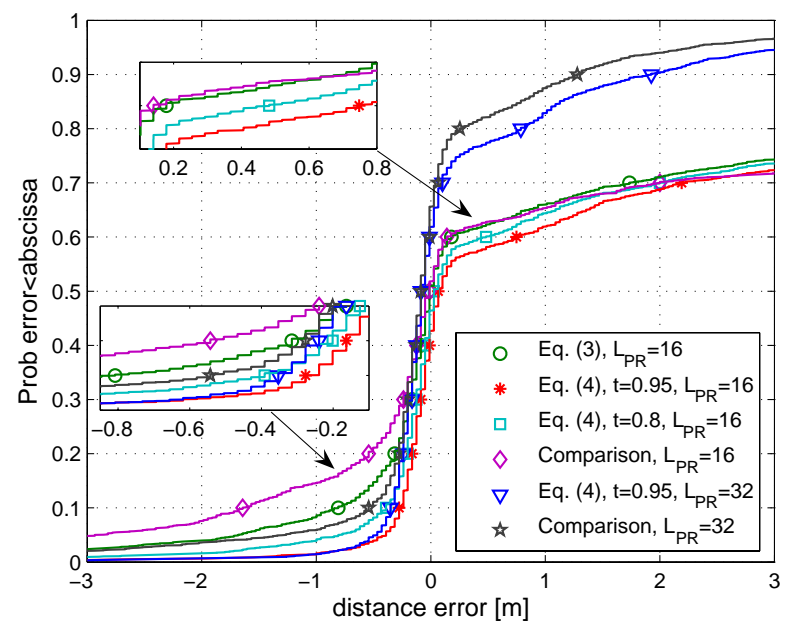

Fig. 1. Example CDF of distance estimation errors

\section{B. Positioning}

Eight beacons and one sensor were randomly placed over a square office space with side $10 \mathrm{~m}$. An additional sensor was placed at the center of the space. The thresholded distance estimator in (4), with a threshold of $t=0.95$, was used and positions were estimated using the POCS algorithms described in Sec. IV. The probability of a LOS channel realization was $P_{\mathrm{LOS}}=0.75$, and a preamble length of $L_{\mathrm{PR}}=32$ was used. The simulations were repeated 500 times, each with new node layout, noise and channel realizations. The results are plotted in Fig. 2. For comparison, a randomly initialized weighted least-squares (WLS) algorithm, proposed for instance in [2] was implemented. The WLS implementation assumed perfect NLOS detection and discarding in one case, and was totally naive to NLOS in the other. A number of NLOS detection algorithms have been proposed in the literature, but none of them is perfect, and so the idea of an NLOS-aware WLS approach is fairly optimistic. The ranging input to both WLS approaches was the estimator in (3) weighted by (5). Using the comparison estimator as input significantly decreased performance in all scenarios where SINR was not high.

The resilience of POCS, especially circular POCS with the centered sensor position, to positive errors induced for instance by NLOS effects is clear. It is also seen that the WLS approach, despite perfect NLOS channel detection, suffers from convergence in local minima [10]. The NLOS-naive WLS of course suffers from considerable performance degradation. The impact of network geometry on performance is noted when comparing results for centered and random sensor location. It is also noted that hybrid POCS outperforms circular POCS when the sensor often is located outside the beacon perimeter, in which case circular POCS is not suitable [6]. If beacons are instead positioned at room corners, performance improves considerably. Due to space limitations, additional simulation results and comparisons have been left out. However, the conclusions drawn above seem valid in all simulated scenarios where the SINR was not very high, in which case ranging estimators converge to the same estimate.

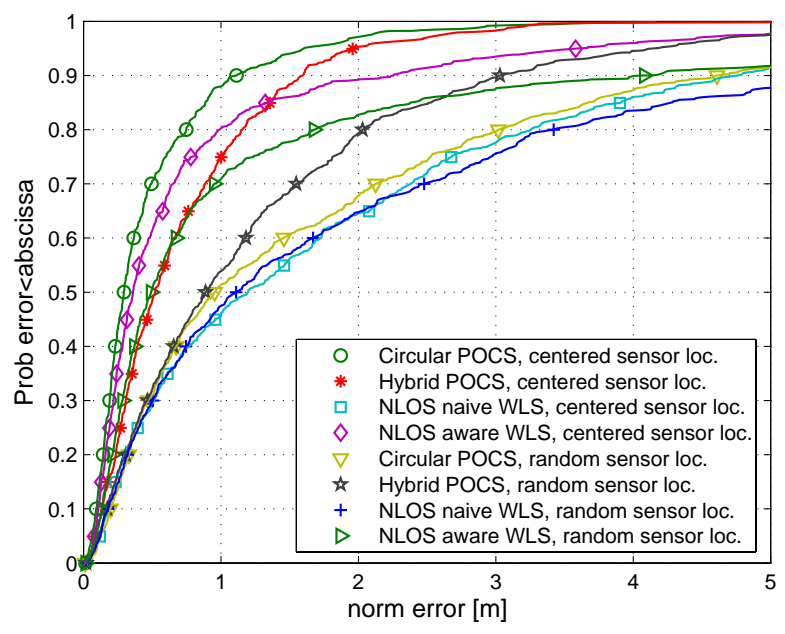

Fig. 2. CDF of position estimation errors

\section{CONCLUSiONS AND FUTURE WORK}

In this work we investigate the advantages of adapting the physical layer distance estimator to the characteristics of a positioning algorithm. With this approach, we obtain a ranging and positioning system that offers low complexity and good performance, while being robust to multipath, NLOS channel effects and least-squares type objective function local minima.

It is the authors opinion that significant performance gains may be achieved by understanding and tuning the predefined parameters, e.g., $P_{\mathrm{FA}}, \lambda$, etc., involved in our proposed approach. Also, the crude $\chi_{2}^{2}$-model of correlator outputs under hypothesis $\mathcal{H}_{1}$ could be improved.

Further studies on the effect of using energy-detector frontends instead of matched filters, having non-homogenous sensor front-ends (matched filter and energy detector front-ends in the same network), and using the distance estimator output when tracking mobile nodes are currently on-going.

\section{REFERENCES}

[1] M. Win and R. Scholtz, "Impulse radio: How it works," in IEEE Comm. Letters, Feb. 1998.

[2] S. Gezici, Z. Tian, et al., "Localization via ultra-wideband radios," IEEE Signal Processing Magazine, vol. 22, pp. 70-84, 2005.

[3] J.-Y. Lee and R. A. Scholtz, "Problems in modeling UWB channels," Proc. Asilomar Conf. on Systems and Computers, vol. 1, pp. 706-711, 2002.

[4] - , "Ranging in a dense multipath environment using an UWB radio link," IEEE Journal on Sel. Areas in Comm., vol. 20, pp. 1677-1683, 2002.

[5] I. Guvenc, Z. Sahinoglu, and P. Orlik, "TOA estimation for IR-UWB systems with different transciever types," IEEE Transactions on Microwave Theory and Techniques, vol. 54, pp. 1876-1886, 2006.

[6] M. Rydström, E. G. Ström, and A. Svensson, "Robust sensor network positioning based on projections onto circular and hyperbolic convex sets (POCS)," in Proc. 7th IEEE workshop on Signal Processing Advances in Wireless Communications, July 2006.

[7] A. Molisch, K. Balakrishnan, et al., "IEEE p802.15-04/662r2-tg4a," IEEE P802.15 WPAN, Tech. Rep., July 2005.

[8] S. M. Kay, Fundamentals of Statistical Signal Processing: Estimation Theory. Prentice Hall PTR, 1993.

[9] Y. Censor and S. A. Zenios, Parallel Optimization, Theory, Algorithms, and Applications. Oxford University press, 1997.

[10] D. Blatt and A. O. Hero, "Sensor network source localization via projection onto convex sets (POCS)," in Proc. 2005 IEEE International Conference on Acoustics, Speech, and Signal Processing, Philadelphia, USA, Mar. 2005. 